\title{
Empreinte parentale et Aide Médicale à la Procréation (AMP)
}

\author{
Céline CHALAS, Pierre JOUANNET
}

Laboratoire de Biologie de la Reproduction, GREFH, Hôpital Cochin-Université René Descartes, Paris

\section{RÉSUMÉ}

La prise en charge de l'infertilité a débuté il y a plus de deux siècles par la réalisation d'inséminations artificielles, technique interférant peu sur le processus de reproduction puisque la fécondation se déroule in vivo. Les années 80 ont vu l'émergence de la fécondation in vitro (FIV) nécessitant une stimulation hormonale soutenue, une fécondation ex vivo et une culture embryonnaire. Enfin, l'arrivée de I'ICSI (Intra Cytoplasmic Sperm Injection) a révolutionné la prise en charge des couples en permettant de créer un embryon par simple microinjection d'un spermatozoïde, même anormal ou immature, dans un ovocyte. Rapidement, la question de l'impact de ces techniques sur la santé des enfants ainsi conçus a été posée. Des études récentes ont suggéré un lien entre aide médicale à la procréation et développement de pathologies d'empreinte. En effet, l'empreinte parentale établie spécifiquement sur chacun des gamètes et transmise à l'embryon peut être sensible à des facteurs externes. La manipulation et la culture in vitro des gamètes et embryons, inhérentes à la réalisation de la FIV/ICSI, pourraient avoir des conséquences sur l'établissement et/ou la conservation de l'empreinte. La connaissance des mécanismes d'acquisition et de maintien de l'empreínte et l'analyse de leur dérégulation sont nécessaires pour évaluer ce risque potentiel.

Mots clés : empreinte parentale, méthylation, pathologies d'empreinte, aide médicale à la procréation

\section{INTRODUCTION}

L'histoire de la fécondation in vitro a commencé en 1978 par la naissance de Louise Brown en Angleterre. Elle a été pratiquée en France dès 1981, date à partir de laquelle le recours aux techniques d'aide médicale à la procréation (AMP) n'a cessé d'augmenter. Un grand pas contre la stérilité a été franchi avec la mise au point de la microinjection intracytoplasmique de spermatozoïde ou ICSI, permettant de créer un embryon, après sélection d'un spermatozoïde et injection, à l'aide d'une micropipette de verre, dans un ovocyte. L'ICSI a permis la prise en charge de nombreux cas d'infertilité masculine, ce d'autant plus que les spermatozoïdes micro-injectés peuvent être isolés d'éjaculats frais ou de sperme congelé ou peuvent être des spermatozoïdes immatures prélevés chirurgicalement dans l'épididyme ou dans le testicule.

Cependant, dès l'introduction de la FIV et surtout depuis celle de l'ICSI, s'est posée la question de l'innocuité de ces méthodes. Des études d'évaluation de l'impact de ces techniques sur les enfants conçus par AMP ont été menées. Des diminutions de poids de naissance $[1,26]$, des augmentations de l'incidence de malformations congénitales [14, 33] et d'aneuploïdies chromosomiques [32], ainsi que des retards de développement psychomoteur ou mentaux ont été rapportés mais les résultats des études statistiques sont discordants et ne mettent pas en évidence de marqueurs ou de risque significatif clairement attribuable à telle ou telle technique d'AMP. Néanmoins, depuis 2002, un nouveau risque lié à la pratique de l'AMP a été évoqué [6], la dérégulation possible de gènes soumis à empreinte suggérée par l'augmentation de patholo-

\section{Correspondance :}

Pr Pierre JOUANNET - Laboratoire de Biologie de la Reproduction, GREFH, Hôpital Cochin, Université Paris V, 123 Boulevard de Port Royal, 75014 Paris, France -

Tel 0158411570 - Fax 0158411565 -

Email pierre.jouannet@cch.ap-hop-paris.fr 
gies d'empreinte chez les enfants conçus par AMP $[7,12$, 22].

Afin de mieux comprendre les raisons possibles de survenue de ces anomalies, chacune des étapes de la FIV/ICISI et leurs éventuelles conséquences sur l'acquisition et/ou le maintien de l'empreinte parentale seront analysées dans cet article.

\section{EMPREINTE PARENTALE}

L'empreinte parentale est une marque épigénétique établie différentiellement sur les deux allèles parentaux au niveau de gènes spécifiques, dits gènes soumis à empreinte (GSE). Ce marquage permet une expression monoalléliques des GSE et définit une empreinte paternelle et maternelle. Différents éléments peuvent caractériser ce marquage : structure de la chromatine, modification des protéines histones, méthylation de l'ADN. Nous nous intéresserons ici essentiellement à la méthylation des cytosines de l'ADN qui est un mécanisme important de l'établissement et du maintien de l'empreinte. Cette méthylation est apposée de façon allèle spécifique au niveau des GSE par une famille d'enzymes, les ADN methyltransférases. Pour que chaque individu, dans ses cellules somatiques, soit porteur d'une empreinte paternelle et maternelle, il faut que les gamètes transmettent une empreinte sexe spécifique et que leur réunion au moment de la fécondation permette de maintenir cet équilibre. Ainsi le spermatozoïde transmet une empreinte uniquement paternelle et l'ovocyte uniquement maternelle (Figure 1). L'empreinte est ensuite conservée au cours du développement embryonnaire puis de la vie adulte.

Les GSE sont essentiels au développement des mammifères. En effet, ils interviennent dans le développement de l'embryon où ils jouent un rôle crucial dans la croissance, le développement psychomoteur et la fonctionnalité du placenta, expliquant que leurs dérégulations, qu'elles soient transmises par les gamètes ou acquises au cours du développement embryonnaire, sont à l'origine de pathologies graves. Par ailleurs, le développement de cancers chez l'enfant et l'adulte peut être associé à des dérégulations d'empreintes $[8,18]$.

\section{AIDE MÉDICALE À LA PROCRÉATION ET PATHOLOGIES D'EMPREINTE}

Entre les années 1990 et 2002, quelques cas sporadiques de pathologies d'empreinte, déclarées chez des enfants conçus par AMP, ont été répertoriés sans conclusion particulière (Tableau 1). Cependant, fin 2002, deux cas de syndrome d'Angelman (AS) après ICSI sont rapportés [4], puis un nouveau en 2003 [25]. Cette même année, trois études américaine, française et anglaise ont rapporté un nombre élevé de cas de syndrome de Beckwith-Wiedemann (BWS) chez des enfants conçus par AMP [7, 12, 22], observation confirmée par une étude rétrospective australienne [13] (Tableau 2).

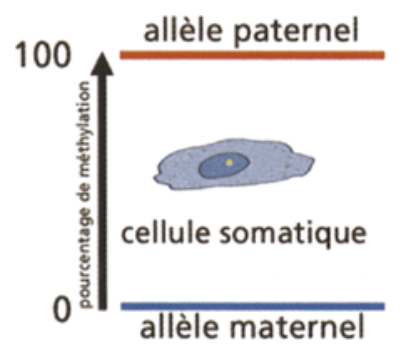

transmission paternelle de l'empreinte
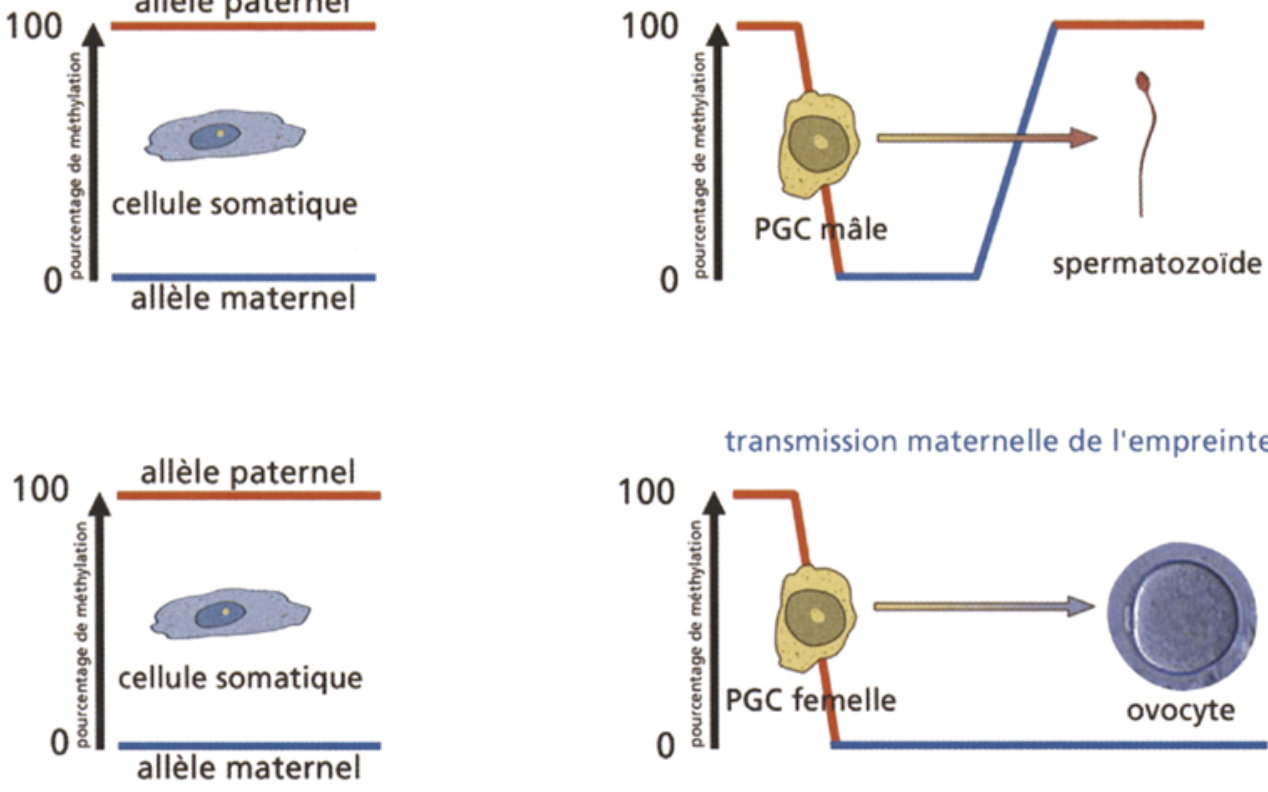

transmission maternelle de l'empreinte

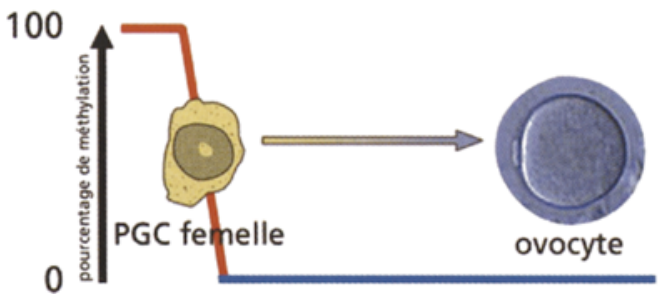

Figure 1 : Acquisition sexe-spécifique de l'empreinte parentale dans les lignées germinales.

L'une des caractéristiques des gènes soumis à empreinte est leur expression monoallélique en fonction de l'origine parentale des allèles. Afin de conserver cette spécificité d'expression dans les cellules somatiques de chaque nouvel individu, il faut que, dans les cellules germinales, l'empreinte soit établie selon le sexe de l'individu et transmise spécifiquement par chaque gamète. Ainsi, le marquage gamétique (épigénotype gamétique) doit être réversible et effaçable. L'effacement des modifications épigénétiques survient dans les cellules germinales primordiales (PGCs). Après cette étape d'effacement, une nouvelle empreinte parentale sexe-spécifique est établie dans les lignées germinales mâles et femelles. 
Tableau 1 : Cas rapportés de pathologies d'empreinte après AMP entre 1990 et 2002.

\begin{tabular}{llccc}
\hline Pathologie & \multicolumn{1}{c}{ Référence } & Nombre de cas & Technique d'AMP & Perte empreinte \\
BWS & Bonduelle et al. [3] & 1 & ICSI & Non déterminé \\
BWS & Boerritger et al. [2] & 1 & ICSI & Non déterminé \\
BWS & Olivennes et al. [24] & 1 & ICSI & Non déterminé \\
BWS & Koudstaal et al. [19] & 1 & FIV & FIV \\
BWS & Sutcliffe et al. [30] & 1 & Nóterminé déterminé \\
\hline
\end{tabular}

Tableau 2 : Cas de Beckwith-Wiedemann recensés après AMP.

\begin{tabular}{|c|c|c|c|c|}
\hline Auteurs & $\begin{array}{l}\text { Nombre total de cas } \\
\text { de BWS étudiés }\end{array}$ & $\begin{array}{l}\text { Nombre des BWS } \\
\text { nés après AMP }\end{array}$ & $\begin{array}{c}\% \text { enfants AMP } \\
\text { dans population générale }\end{array}$ & $\begin{array}{l}\text { Risque de BWS } \\
\text { si AMP }\end{array}$ \\
\hline Halliday et al. [13] & 37 & $4(10,8 \%)$ & & $\times 9$ \\
\hline DeBaun et al. [7] & 65 & $3(4,6 \%)$ & $0,76 \%$ & $\times 5$ \\
\hline Maher et al. [22] & 149 & $6(4,0 \%)$ & $1,0 \%$ & $\times 4$ \\
\hline Gicquel et al. [12] & 149 & $6(4,0 \%)$ & $1,3 \%$ & $\times 3$ \\
\hline
\end{tabular}

Le syndrome d'Angelman est un retard mental sévère, diagnostiqué précocement, associé à une ataxie et une hypotonie et causé par la perte d'empreinte du gène UBE3A. Le BWS est un syndrome de croissance excessive associant plusieurs malformations, tels que macroglossie, omphalocèle, une fréquence élevée de diabète néonatal et surtout un risque augmenté de développement de tumeurs dans l'enfance, en particulier de tumeur de Wilm. Plusieurs GSE de la région chromosomique 11p15.5, H19, IGF2, Kcnq1ot et CDNK1 sont impliqués dans ce syndrome. Alors que les cas d'AS observés restent isolés, les cas rapportés de BWS sont plus nombreux (4 à 6 cas par étude). Dans les différentes études où des analyses géniques ont été faites, la même anomalie a été trouvée dans tous les cas. Elle concerne le gène Kcnq1ot seulement dans les cas de BWS et UBE3A dans les cas de AS.

Par ailleurs, aussi bien pour le BWS que pour le AS, l'anomalie correspond à une perte de l'empreinte maternelle entraînant une expression biallélique du GSE paternel. Le mécanisme de perte d'empreinte, qui est le plus fréquemment responsable du développement du BWS, n'est que très rarement impliqué dans le $A S$, ce qui amène à penser qu'un facteur inhérent à la pratique de l'AMP favorise cette perte d'empreinte maternelle. Aucune technique particulière d'AMP n'a pu être mise en cause jusqu'à présent. En effet, toutes les techniques disponibles ont été utilisées pour la conception des enfants atteints des pathologies d'empreinte décrites : spermatozoïdes congelés ou non, provenant d'éjaculat ou prélevés chirurgicalement dans l'épididyme ou le testicule, fécondation par FIV ou ICSI, transfert d'embryons congelés ou non.

La survenue de BWS chez les enfants conçus par AMP est à rapporter aux observations faites chez les animaux issus de FIV et décrites sous "syndrome du gros veau " (Loss Off Imprinting, LOS). Dès 1998, des anomalies touchant les chèvres et les moutons avaient été répertoriées après simple culture d'embryons in vitro ou FIV/ICSI. Les animaux concernés présentaient un poids de naissance élevé, des malformations, un diabète néonatal et une forte mortalité dans les premières années de vie [35]. Ces manifestations ont été partiellement corrélées à la dérégulation d'un GSE, igf 2 , qui comme dans la pathologie humaine correspond à une perte de l'empreinte maternelle [34].

Néanmoins, les données connues actuellement ne permettent pas d'exclure une contribution paternelle au développement d'anomalies d'empreinte pas plus qu'elles ne permettent de remettre en cause la pratique de la $\mathrm{FIV} / \mathrm{ICISI}$, le nombre de cas rapportés restant très faible par rapport aux nombre d'enfants nés depuis les années 80 , qui se comptent par centaines de milliers.

Afin de mieux évaluer l'existence potentielle d'un risque épigénétique au cours de la prise en charge en AMP, il est nécessaire de mieux comprendre comment l'empreinte est établie et maintenue chez les gamètes et l'embryon et quelle influence pourrait avoir la FIV sur son altération éventuelle.

\section{EMPREINTE PARENTALE DANS LES GAMĖTES : ACQUISITION, MAINTIEN ET INTER- FÉRENCES POSSIBLES DES PRATIQUES D'AMP}

L'empreinte est transmise de façon sexe spécifique au nouvel individu par l'intermédiaire des gamètes. Pour cela, l'empreinte est effacée précocement au niveau des cellules germinales primordiales puis rétablie spécifiquement dans chacune des deux lignées germinales mâles et femelles.

\section{Lignée germinale mâle}

L'étape d'établissement de l'empreinte sexe spécifique dans la lignée germinale mâle reste controversée. Les étu- 
des menées chez la souris [5] et l'homme [17] ont établi, pour $H 19$, une acquisition précoce de la méthylation dès le stade spermatogonie et le maintien de cette méthylation tout au long de la différenciation de la lignée germinale. Cependant, chez la souris, il a également été rapporté une acquisition plus tardive de l'empreinte au stade spermatocyte-spermatide [31].

Au stade spermatozoïde, H19, MEST [17] SNRPN [11] chez l'homme et Mest, Snrpn, H19, peg 3, igf2r chez la souris [21] ont une empreinte conforme au sexe transmetteur en faveur d'une acquisition précoce de l'empreinte. Néanmoins, la question d'une empreinte acquise dans les spermatozoïdes, pour l'ensemble des gènes soumis à empreinte, reste posée. Ainsi, El-maari et al. ont démontré une acquisition définitive de l'empreinte de SNRPN dans les gamètes mâles au moment de la fécondation ou après [10] (Figure 2). Le risque de transmission d'anomalies épigénétiques lors de l'utilisation de gamètes mâles en AMP reste donc posé, d'autant plus lors de l'utilisation de gamètes immatures, spermatozoïdes testiculaires ou épididymaires prélevés chirurgicalement, sur lesquels peu d'études ont été menées.

Par ailleurs, le rôle de la cryoconservation, soit du cryoprotecteur et/ou de la technique de congélation elle même sur le maintien de l'empreinte reste à déterminer, aucune analyse n'ayant été réalisée.

\section{Lignée germinale femelle}

L'acquisition de l'empreinte dans la lignée femelle apparaît plus complexe car multi-étapes. En effet, deux études récentes, menées chez la souris [20,23], ont rapporté une acquisition progressive de l'empreinte au fur et à mesure de la croissance ovocytaire. Cet établissement progressif implique que l'ensemble des gènes étudiés n'acquière leur empreinte qu'au stade de vésicules germinatives issues de follicules antraux (Figure 3). Chez l'homme, une étude menée dans des ovocytes bloqués en métaphase de 1 ère et $2^{\text {ème }}$ division a pareillement trouvé une méthylation normale du gène SNRPN [11], alors q'une étude contradictoire, menée également sur vésicules germinatives, a rapporté une acquisition de la méthylation du gène SNRPN audelà du stade métaphase II, soit à la fécondation ou juste après [10].

La croissance ovocytaire est corrélée à la synthèse enzymatique et en particulier à celle des methyltransférases, indispensables à l'apposition des groupements méthyles sur les cytosines des GSE. L'acquisition de la méthylation au fur et à mesure de la maturation ovocytaire fait craindre une interférence entre établissement de l'empreinte et stimulation hormonale lors de l'AMP. En effet, une croissance forcée, sous l'action des gonadotrophines, pourrait influer d'une part sur l'acquisition de l'empreinte des gènes tardi-

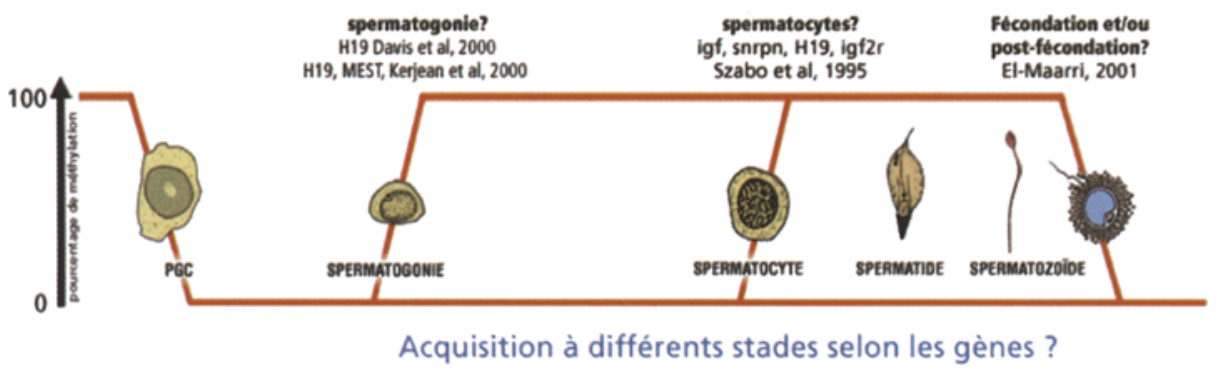

Figure 2 : Hypothèses sur le stade cellulaire d'acquisition de l'empreinte d'un gène méthylé au cours de la différenciation et de la maturation de la lignée germinale mâle. Plusieurs études se sont intéressées, aussi bien chez l'homme que chez la souris, au stade cellulaire germinal d'acquisition de l'empreinte. Plusieurs observations rapportent soit une acquisition précoce soit une acquisition tardive et peu de gènes ont été étudiés. Ainsi, le stade précis d'acquisition de l'empreinte ainsi que la cinétique d'acquisition en fonction des gènes soumis à empreinte restent controversés.

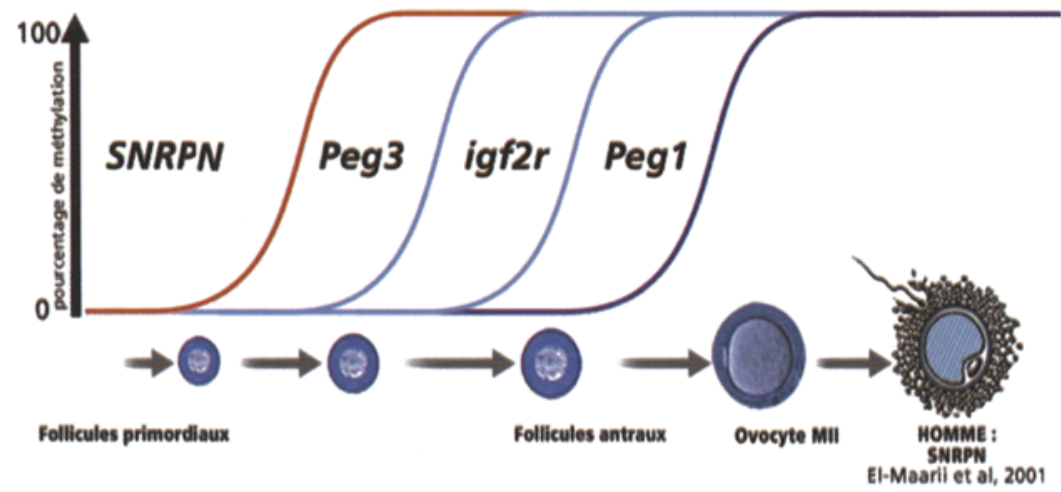

Figure 3 : Acquisition de l'empreinte parentale gamète spécifique dans la lignée germinale femelle. Dans l'ovocyte, il y a acquisition de l'empreinte au fur et à mesure de la croissance ovocytaire. L'acquisition est précoce pour certains gènes, dès le stade follicule secondaire pour SNRPN, tandis qu'elle est plus tardive pour d'autre, tel que le stade follicule antral pour peg1. 
vement "imprintés " et d'autre part sur la synthèse des méthyltransférases. Cette éventualité est d'autant plus considérée que dans tous les cas de pathologies d'empreinte rapportés, il s'agit systématiquement d'une dérégulation maternelle de l'empreinte. Des études sur modèles animaux sont nécessaires afin d'évaluer ce phénomène.

La question parait également posée avec le récent avènement de la maturation in vitro (MIV) qui consiste à faire maturer in vitro des ovocytes immatures prélevés au stade vésicule germinative ou métaphase I jusqu'au stade métaphase II, impliquant une culture prolongée des ovocytes et donc l'influence possible de facteurs externes. La très récente introduction de cette technique en AMP ne permet pas encore d'évaluer toutes ses conséquences. Enfin, dans le futur, l'utilisation du tissu ovarien cryoconservé sera confronté aux mêmes interrogations. En effet, actuellement, une cryconservation de tissu ovarien est proposée à des femmes jeunes devant subir un traitement gonadotoxique. On espère un jour avoir les moyens de faire maturer in vitro les follicules issus de ce tissu afin de permettre à ces femmes de procréer. Cette situation impliquera un temps de culture très prolongé. Chez la souris, après maturation des follicules préantraux issus d'ovaires de souris jusqu'au stade follicules antraux en 11 jours de culture, l'étude de la méthylation de trois GSE, H19, mest et igf $2 r$, a mis en évidence des dérégulations de méthylation concernant les trois gènes [16]. Une connaissance approfondie des conséquences de la culture sur l'empreinte humaine et de l'influence de facteurs externes sur cette empreinte paraît donc indispensable avant que ces techniques ne soient appliquées à large échelle chez l'humain.

\section{EMBRYON PRÉ-IMPLANTATOIRE : EMPREINTE PARENTALE ET PRATIQUES D'AMP}

Très peu d'études se sont intéressées au statut de méthylation des GSE de l'embryon préimplantatoire. Seuls Geuns et al. ont rapporté une méthylation de $50 \%$ du gène SNRPN dans des embryons préimplantatoires humains conçus par FIVIICSI suggérant d'une part un équilibre normal entre l'absence de méthylation paternelle $(0 \%)$ et $100 \%$ de méthylation maternelle et d'autre part aucune interférence de l'ICSI sur l'acquisition de la méthylation de ce gène [11]. Cette étude isolée reste à confirmer à plus grande échelle.

Au contraire, des études de méthylation globale de l'ADN, menées chez la souris, ont montré des anomalies de méthylation, chez les embryons conçus par FIV ou après simple stimulation hormonale de la femelle, versus des embryons conçus in vivo [27]. Ces différences de méthylation ont été corrélées à des anomalies de développement embryonnaire impliquant un lien direct entre AMP et dérégulation. Cependant, la complexité de l'expression des GSE dans l'embryon et leur grande tissu spécificité rend toute analyse très difficile, de sorte que l'on ne peut se prononcer, à l'heure actuelle, sur la dérégulation embryonnaire des GSE consécutive à l'AMP.

\section{INFLUENCE DE LA CULTURE IN VITRO SUR LE MAINTIEN DE L'EMPREINTE ET SA DÉRÉGULATION}

Les effets de la culture sur les individus à naître ont rapidement été constatés chez les veaux et les brebis par le développement du LOS [35]. Ce syndrome se développe essentiellement chez des animaux issus d'embryons et/ou d'ovocytes exposés à plusieurs jours de culture, d'autant plus que le milieu a été supplémenté en sérum et qu'il y a eu co-culture. Les effets phénotypiques de la culture ont rapidement été démontrés en constatant des diminutions du développement des embryons en morulas et blastocytes in vitro versus in vivo chez la chèvre, des gestations plus longues et des poids de naissance globalement augmentés chez les ovins et caprins [15, 28]. La certitude d'une contribution épigénétique à ces phénomènes a été démontrée chez la chèvre, où l'empreinte de igf $r$ a été retrouvée anormale chez les animaux malades issus de FIV [34]. II n'existe pas de données humaines claires sur ce problème si ce n'est les quelques cas de pathologies d'empreinte rapportés pour lesquelles le niveau de dérégulation pourrait se situer dans le gamète femelle ou au cours de la culture voir ultérieurement. II semble cependant qu'une durée de culture supérieure à 5 jours et la composition du milieu de culture favorisent des dérégulation d'empreinte chez la souris et la brebis $[9,28]$. Ainsi, le rôle de la culture se pose en cas de transfert de blastocystes, même si aucune anomalie humaine n'a encore été répertoriée, comme dans le cas de la culture ovocytaire prolongée évoquée précédemment.

\section{CONCLUSION}

Au total, de nombreux facteurs sont capables d'interférer avec l'établissement ou le maintien de l'empreinte parentale à chacune des étapes mises en jeu dans la réalisation des FIV/ICSI, soit dès qu'il y a manipulation de gamètes et/ou d'embryons (Figure 4). Cependant, le faible nombre d'études menées dans l'espèce humaine et les résultats parfois contradictoires des travaux de recherche entre eux ne permettent pas de conclure sur le rôle de ces techniques. II apparaît donc nécessaire de développer des protocoles de recherche sur gamètes et embryons et d'y associer un suivi des grossesses et des naissances. II semble également important de poursuivre ce suivi à l'âge adulte, des données récentes suggérant que certaines anomalies d'empreinte se manifesteraient à l'âge adulte sous forme de susceptibilité au développement de cancers, diabètes ou pathologies cardio-vasculaires [22]. Ce type de risque ne peut être évalué chez les enfants conçus par AMP, les plus vieux n'ayant qu'une vingtaine d'années, mais nécessiterait sûrement d'être vigilants.

Les données actuelles sont rassurantes car le nombre d'anomalies liées à un désordre d'empreinte répertorié à la naissance reste non significatif, ce qui ne doit pas retarder la mise en place d'études simples d'évaluation du risque épigénétique en AMP. 


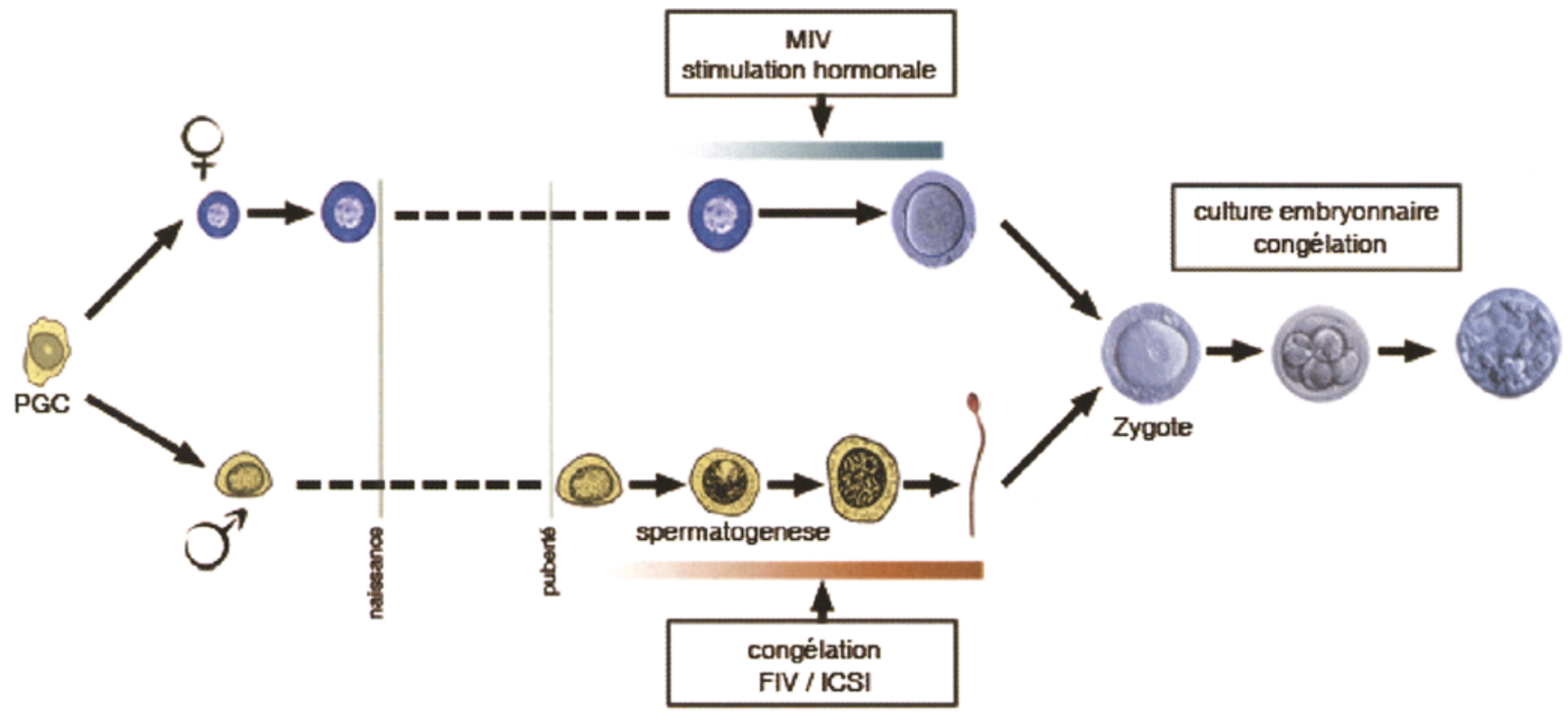

Figure 4 : Niveaux possibles de dérégulations de l'empreinte des gènes soumis à empreinte au cours des différentes étapes techniques mises en jeu par la pratique de I'AMP. Les niveaux de dérégulations d'empreinte des gènes par les pratiques de FIV et d'ICSI sont possibles à toutes les étapes de la FIVIICSI sans qu'aucun n'ait été confirmé ou infirmé. Les conséquences de ces dérégulations restent encore à mesurer aussi bien chez les enfants à naître que chez les adultes ainsi conçus

\section{REFERENCES}

1. BERGH T., ERICSON A., HILLENSJO T., NYGREN K. G., WENNERHOLM U.B. : Deliveries and children born after invitro fertilisation in Sweden 1982-95: a retrospective cohort study. Lancet, 1999, 354 : 1579-1585.

2. BOERRIGTER P.J., DE BIE J.J., MANNAERTS B.M., VAN LEEUWEN B.P., PASSIER-TIMMERMANS D.P. : Obstetrical and neonatal outcome after controlled ovarian stimulation for IVF using the GnRH antagonist ganirelix. Hum. Reprod,, 2002, $17: 2027-2034$.

3. BONDUELLE M., LIEBAERS I., DEKETELAERE V. et al. : Neonatal data on a cohort of 2889 infants born after ICSI (1991-1999) and of 2995 infants born after IVF (1983-1999). Hum. Reprod., 2002, $17: 671-694$.

4. COX G., BURGER J., LIP V. et al. : Intracytoplasmic sperm injection may increase the risk of imprinting defects. Am. J. Hum. Genet., 2002, $71: 162-164$.

5. DAVIS T.L., YANG G.J., MCCARREY J.R., BARTOLOMEI M.S. : The $\mathrm{H} 19$ methylation imprint is erased and re-established differentially on the parental alleles during male germ cell development. Hum. Mol. Genet., 2000, 9 : 2885-2894.

6. DE RYCKE M., LIEBAERS I., VAN STEIRTEGHEM A. : Epigenetic risks related to assisted reproductive technologies : risk analysis and epigenetic inheritance. Hum. Reprod., 2002, 17 : 2487-2494.

7. DEBAUN M.R., NIEMITZ E.L., FEINBERG A.P. : Association of in vitro fertilization with Beckwith-Wiedemann syndrome and epigenetic alterations of LIT1 and H19. Am. J. Hum. Genet., 2003, 72 : 156-160.

8. DEBAUN M.R., NIEMITZ E.L., MCNEIL D.E. et al. : Epigenetic alterations of $\mathrm{H} 19$ and LIT1 distinguish patients with BeckwithWiedemann syndrome with cancer and birth defects. Am. J. Hum. Genet., 2002, 70 : 604-611.
9. DOHERTY A.S., MANN M.R., TREMBLAY K.D., BARTOLOMEI M.S., SCHULTZ R. : M. Differential effects of culture on imprinted $\mathrm{H} 19$ expression in the preimplantation mouse embryo. Biol. Reprod., 2000, 62 : 1526-1535.

10. EL-MAARRI O., BUITING K., PEERY E.G., et al. : Maternal methylation imprints on human chromosome 15 are established during or after fertilization. Nat. Genet., 2001, $27: 341$ 344.

11. GEUNS E., DE RYCKE M., VAN STEIRTEGHEM A., LIEBAERS I. : Methylation imprints of the imprint control region of the SNRPN-gene in human gametes and preimplantation embryos. Hum. Mol. Genet., 2003, 12 : 2873-2879.

12. GICQUEL C., GASTON V., MANDELBAUM J. et al. : In vitro fertilization may increase the risk of Beckwith-Wiedemann syndrome related to the abnormal imprinting of the KCN1OT gene. Am. J. Hum. Genet., 2003, 72 : 1338-1341.

13. HALLIDAY J., OKE K., BREHENY S., ALGAR E., BECKWITHWIEDEMANN DJ.A. : syndrome and IVF : a case-control study. Am. J. Hum. Genet., 2004, $75:$ 526-528.

14. HANSEN M., KURINCZUK J.J., BOWER C., WEBB S. : The risk of major birth defects after intracytoplasmic sperm injection and in vitro fertilization. N. Engl. J. Med., 2002, 346 : 725730.

15. HOLM P., WALKER S.K., SEAMARK R.F. : Embryo viability, duration of gestation and birth weight in sheep after transfer of in vitro matured and in vitro fertilized zygotes cultured in vitro or in vivo. J. Reprod. Fertil., 1996, $107: 175-181$.

16. KERJEAN A., COUVERT P., HEAMS T. et al. : In vitro oocyte growth in mice affects maternal primary imprinting establishment. Eur. J. Hum. Genet., 2003, 11 : 493-496.

17. KERJEAN A., DUPONT J.M., VASSEUR C. et al. : Establishment of the paternal methylation imprint of the human $\mathrm{H19}$ and MEST/PEG1 genes during spermatogenesis. Hum. Mol. Genet., 2000, $9:$ 2183-2187. 
18. KLEIN G. : Epigenetics : surveillance team against cancer. Nature, 2005, $434: 150$.

19. KOUDSTAAL J., BRUINSE H.W., HELMERHORST F.M. et al.: Obstetric outcome of twin pregnancies after in-vitro fertilization: a matched control study in four Dutch university hospitals. Hum. Reprod., 2000, $15: 935-940$.

20. LUCIFERO D., CHAILLET J.R., TRASLER J.M. : Potential significance of genomic imprinting defects for reproduction and assisted reproductive technology. Hum. Reprod. Update, $2004,10: 3-18$.

21. LUCIFERO D., MERTINEIT C., CLARKE H.J., BESTOR T.H., TRASLER J.M. : Methylation dynamics of imprinted genes in mouse germ cells. Genomics, 2002, $79: 530-538$.

22. MAHER E.R., AFNAN M., BARRATT C.L. : Epigenetic risks related to assisted reproductive technologies : epigenetics, imprinting, ART and icebergs ? Hum. Reprod., 2003, 18 : 2508-2511.

23. OBATA Y., KONO T. : Maternal primary imprinting is established at a specific time for each gene throughout oocyte growth. J. Biol. Chem., 2002, $277:$ 5285-5289.

24. OLIVENNES F., MANNAERTS B., STRUIJS M., BONDUELLE M., DEVROEY P. : Perinatal outcome of pregnancy after $\mathrm{GnRH}$ antagonist (ganirelix) treatment during ovarian stimulation for conventional IVF or ICSI : a preliminary report. Hum. Reprod,, 2001, $16:$ 1588-1591

25. ORSTAVIK K.H., EIKLID K., VAN DER HAGEN C.B. et al. : Another case of imprinting defect in a girl with Angelman syndrome who was conceived by intracytoplasmic semen injection. Am. J. Hum. Genet., 2003, 72 : 218-219.

26. SCHIEVE L.A., MEIKLE S.F., FERRE C. et al. : Low and very low birth weight in infants conceived with use of assisted reproductive technology. N. Engl. J. Med., 2002, 346 : 731737.

27. SHI W., HAAF T. : Aberrant methylation patterns at the twocell stage as an indicator of early developmental failure. Mol. Reprod. Dev., 2002, $63:$ 329-334.

28. SINCLAIR K.D., MCEVOY T.G., MAXFIELD E.K. et al. : Aberrant fetal growth and development after in vitro culture of sheep zygotes. J. Reprod. Fertil., 1999, 116 : 177-186.

29. STROMBERG B., DAHLQUIST G., ERICSON A. et al. : Neurological sequelae in children born after in-vitro fertilisation: a population-based study. Lancet, 2002, $359: 461-465$.

30. SUTCLIFFE A.G., D'SOUZA S.W., CADMAN J. et al. : Minor congenital anomalies, major congenital malformations and development in children conceived from cryopreserved embryos. Hum. Reprod., 1995, 10 : 3332-3337.

31. SZABO P.E., MANN J.R. : Biallelic expression of imprinted genes in the mouse germ line: implications for erasure, establishment, and mechanisms of genomic imprinting. Genes Dev., 1995, $9: 1857-1868$.

32. VAN STEIRTEGHEM A., BONDUELLE M., DEVROEY P., LIEBAERS I. : Follow-up of children born after ICSI. Hum. Reprod. Update, 2002, 8 : 111-116.

33. WENNERHOLM U.B., BERGH C., HAMBERGER L. et al. : Incidence of congenital malformations in children born after ICSI. Hum. Reprod., 2000, 15 : 944-948.

34. YOUNG L.E., FERNANDES K., MCEVOY T.G. et al. : Epigenetic change in IGF2R is associated with fetal overgrowth after sheep embryo culture. Nat. Genet, 2001, $27: 153-154$.

35. YOUNG L.E., SINCLAIR K.D., WILMUT I. : Large offspring syndrome in cattle and sheep. Rev. Reprod., 1998, 3 : 155163.
Prix du meilleur DESS (Biologie de la Reproduction) SALF 2004

Manuscrit reçu : juin 2005; accepté juillet 2005.

\section{ABSTRACT}

\author{
Parental imprinting and Assisted Reproductive \\ Technology (ART)
}

\section{Céline CHALAS, Pierre JOUANNET}

Medical intervention in procreation is not recent, as the first artificial insemination (Al) was performed more than two centuries ago. However, the interference in the reproductive process with Al is limited. The first major change concerned the possibility of fertilizing oocytes in vitro (IVF) and culture of preimplantation embryos before their transfer to the uterus. In the early nineties, it was shown that direct injection of a single spermatozoon, even abnormal or immature, into an oocyte could result in a viable embryo and child. These techniques expanded very rapidly and $\mathbf{4 5 , 0 0 0}$ IVFs, with ICSI in $\mathbf{5 0 \%}$ of cases, were performed in France in 2001 (FIVNAT). Although a high incidence of major defects has not been reported, the health status of children born by these techniques is a growing concern. Congenital malformations [Hansen et al., 2002], chromosomal abnormalities [Van Steirteghem et al., 2002], neurological disorders [Stromberg et al., 2002] and low birth weight [Schieve et al., 2002] have been observed and discussed, but none of them seems to be statistically much more frequent after assisted reproductive technology (ART). It is important to determine the mechanism of these defects in order to prevent them. These risks may be related to the parents' health status and to their infertility, but they could also be linked to the techniques used for procreation. Recently, several human and animal studies have suggested an increased risk of imprinting disorders in ART offspring [Debaun et al., 2003; Gicquel et al., 2003; Maher et al., 2003; Halliday et al., 2004]. Several elements can be considered to be responsible for these defects and each step of reproductive technology could be concerned and must be studied. Priority should be given to confirm the incidence of rare genomic imprinting diseases, such as Beckwith Wiedemann Syndrome and Angelman Syndrome after ART. Should systematic analysis of the methylation status of several imprinted genes therefore be performed to evaluate the respective influence of the use of immature gametes, ovarian stimulation and embryo culture involved in IVF/ICSI?

It would also be important to evaluate other epigenetic modifications to determine the role of epigenetic deregulations that could be related to ART.

Key words : parental imprinting, methylation, imprinting diseases, ART 